\title{
Wave function of the radion with BPS branes
}

\author{
G. Cynolter ${ }^{1} 2$ \\ Institute of Theoretical Physics, ETH \\ CH-8093 Zürich, Switzerland
}

\begin{abstract}
We investigate coupled gravity and a bulk scalar field on a slice of $\mathrm{AdS}_{5}$ bulk with special BPS branes at the two ends. With the special scalar potentials on the branes the scalar field does not stabilize the size of the orbifold. With a careful treatment of the general coordinate invariance the complete tensor-scalar spectrum is presented. There are two massless zero modes in the scalar sector, the radion and a dilatonic zero mode. The scalar KK modes acquire masses at the order of the warped mass scale. The four dimensional effective action is of tensor-scalar type.
\end{abstract}

\section{Introduction}

Recently there has been a growing interest in extra dimensional models where the standard model fields localized on a brane. Randall and Sundrum proposed a scenario (RS1) with one extra dimension, two branes of opposite tension at the orbifold points of an AdS bulk [1]. This setup was motivated by the recent progress in string/M-theory and can be realized in string theory [2], [3]. Poincare invariant solution requires a finetuning of the bulk cosmological constant $\Lambda=-\frac{6 k^{2}}{\kappa^{2}}$ and the brane tensions $\lambda_{\text {Planck }}=-\lambda_{\mathrm{TeV}}=\frac{6 k}{\kappa^{2}}$. The exponentially warped metric can generate the hierarchy between the Planck and the electroweak scale with moderate value parameters $k r_{c} \sim \mathcal{O}(40)$. However, there is an exact solution for any $r_{c}$ distance of the two branes. A change in $r_{c}$ is described by a four dimensional massless scalar field, the radion [4]. It couples to matter like a Brans-Dicke scalar and must have a large Brans-Dicke parameter or a mass to recover standard 4-dimensional gravity [5], [6].

Goldberger and Wise proposed a mechanism with a massive bulk scalar field to stabilize the radion [7] . They added special steep $\lambda_{i}(\Phi)$ brane potentials. The competition between the kinetic and potential terms generates a minimum in the effective potential and determines $r_{c}$. Approaches based on naive ansatz without including the backreaction found that the radion acquires an $\mathcal{O}(\mathrm{TeV})$ mass and the couplings to standard model fields are $\sim 1 / \mathrm{TeV}[5]$. Effective field theory calculation gave the same radion mass in [8]. Tanaka, Montes [9] and later independently Csáki et al. included the backreaction and derived a single equations for the scalar degrees of freedom based on Lorentz covariance of the linear perturbations [10]. The equation together with the boundary conditions at the branes defines a hermitic

\footnotetext{
${ }^{1}$ Leave on absence from HAS Research Group for Theoretical Physics, Budapest

${ }^{2}$ E-mail: cynoter@itp.phys.ethz.ch
} 
operator only in special cases, like the stiff potential limit, when $\frac{\partial^{2} \lambda_{i}}{\partial \Phi^{2}} \rightarrow \infty$. In this limit the physical results agree with the naive calculations.

Another mathematically well defined case is when the brane potential is equivalent to the superpotential that generates the bulk scalar potential (13), we call these BPS branes. This type of models with exponential potentials can possibly arise from the bosonic sector of dimensionally reduced supergravity theory [11]. In this letter we give the complete spectrum of linear perturbations around an exact background solution of two flat BPS branes in 5 dimensions. This scenario was analyzed in [12] using two different Gaussian normal coordinate patches with respect to the two branes following the method of [4]. They discussed only one scalar perturbation the massless radion. Solving the equations with two flat branes we find still one more massless dilatonic scalar and the KK tower for the scalar field. This model does not solve the moduli problem but the complete perturbative spectrum can be understood, the limit to the Randall-Sundrum case is smooth and we hope that it gives help to solve the general case with detuned brane potentials. The low energy effective theory is of scalar-tensor nature and generally it cannot be accepted by phenomenology and cosmology.

The paper is organized as follows. In section 2. we present the setup and discuss the background solution using the technique of [13]. In section 3. we consider the simple limit of the Randall Sundrum scenario with an additional bulk scalar field. In section 4 . we fix the gauge, solve the linearized equations and present the complete spectrum and we conclude in the last section.

\section{The model}

In a 5 dimensional $\left(x^{a}=\left(x^{\mu}, y=x^{5}\right)\right) M_{4} \times S_{1} / Z_{2}$ manifold, there are scalar field, gravity and two flat branes at the orbifold fixed points $y=0, r_{c}$. Latin indices run from 1..5 and greek indices $1 . .4$ and prime denotes the derivation with respect to the proper coordinate $y$. 4-dimensional greek indices raised and lowered by $\eta_{\mu \nu}$. The action we consider is

$$
S=\int d^{5} x \sqrt{g}\left(-M^{3} R+\frac{1}{2} g^{a b} \nabla_{a} \Phi \nabla_{b} \Phi-V(\Phi)\right)-\sum_{i=P, T} \int d^{4} x \sqrt{g_{i}} \lambda_{i}(\Phi),
$$

$g_{i}$ is the induced metric on the brane, $\kappa^{2}=1 /\left(2 M^{3}\right)$ is the $5 \mathrm{D}$ cosmological constant, the integration understood on the orbifold.

We want to investigate perturbations around a background solution. The ansatz for solving the Einstein scalar coupled system that respects Lorentz invariance is

$$
\begin{aligned}
d s^{2} & =a^{2}(y) \eta_{\mu \nu} d x^{\mu} d x^{\nu}-d y^{2} \\
\Phi(x, y) & =\Phi_{0}(y) .
\end{aligned}
$$

The warp factor $a(y)$ and $\Phi_{0}(y)$ are continuous even functions. The first derivatives may have jumps, the second derivatives delta singularities at the branes. We use the following form of the Einstein equations

$$
R_{a b}=\kappa^{2} \tilde{T}_{a b}=\kappa^{2}\left(T_{a b}-\frac{1}{3} g_{a b} g^{c d} T_{c d}\right)
$$


The gravitational and scalar equations are the following.

$$
\begin{aligned}
4\left(\frac{a^{\prime}}{a}\right)^{2}+\left(\frac{a^{\prime}}{a}\right)^{\prime} & =-\frac{2 \kappa^{2}}{3} V\left(\Phi_{0}\right)-\frac{\kappa^{2}}{3} \sum_{i} \lambda_{i} \delta\left(y-y_{i}\right) \\
\left(\frac{a^{\prime}}{a}\right)^{2} & =-\frac{\kappa^{2}}{6} V\left(\Phi_{0}\right)+\frac{\kappa^{2}}{12} \Phi_{0}^{\prime 2} \\
\Phi_{0}^{\prime \prime} & =-4 \frac{a^{\prime}}{a} \Phi_{0}^{\prime}+\frac{\partial V\left(\Phi_{0}\right)}{\partial \Phi}+\sum_{i} \frac{\partial \lambda_{i}}{\partial \Phi} \delta\left(y-y_{i}\right)
\end{aligned}
$$

The boundary conditions are

$$
\left[\frac{a^{\prime}}{a}\right]_{y_{i}}=-\frac{\kappa^{2}}{3} \lambda_{i}, \quad\left[\Phi_{0}^{\prime}\right]_{y i}=\frac{\partial \lambda_{i}}{\partial \Phi} .
$$

With $Z_{2}$ symmetry and working in the $\left(0, r_{c}\right)$ interval the jump is twice the value of the derivative calculated from the inside of the interval and there is sign difference at the two branes.

An analytic solution of these equation is generated by the technique proposed by DeWolfe et al.[13]. With one $W(\Phi)$ superpotential a flat solution is generated via

$$
\begin{aligned}
\Phi_{0}^{\prime} & =\frac{1}{2} \frac{\partial W}{\partial \Phi} \\
\frac{a^{\prime}}{a} & =-\frac{\kappa^{2}}{6} W\left(\Phi_{0}\right), \\
V\left(\Phi_{0}\right) & =\frac{1}{8}\left(\frac{\partial W\left(\Phi_{0}\right)}{\partial \Phi}\right)^{2}-\frac{\kappa^{2}}{6} W\left(\Phi_{0}\right)^{2} .
\end{aligned}
$$

These solve the equations of motions if the boundary conditions for $W(\Phi)$ are fulfilled ( + for $y=0$ and - for $y=r_{c}, \Phi_{i}=\Phi_{0}\left(y_{i}\right)$ ).

$$
\begin{aligned}
\lambda_{i}\left(\Phi_{i}\right) & = \pm W\left(\Phi_{i}\right) \\
\frac{\mathrm{d} \lambda_{i}}{\mathrm{~d} \Phi}\left(\Phi_{i}\right) & = \pm \frac{\mathrm{d} W}{\mathrm{~d} \Phi}\left(\Phi_{i}\right)
\end{aligned}
$$

Exactly one fine tuning is needed to find a flat background solution. There is no need for supersymmetry to find a solution though the bulk plus brane action completely vanishes substituting the solution. This technique reduces the coupled system of differential equation to decoupled ordinary differential equations and can be solved (generally numerically) for arbitrary $V(\Phi)$ and $\lambda_{1}(\Phi)$ tuning one additive constant in $\lambda_{2}(\Phi)$. Explicit solution can be constructed defining first $W(\Phi)$, like $W(\Phi)=c-u \Phi^{2}$ or $W(\Phi)=e^{\alpha \Phi}$.

We will investigate perturbations in models where the fine tuning of the scalar brane- and superpotential (12) is valid at all order for arbitrary $\Phi$.

$$
\lambda_{i}(\Phi)= \pm W(\Phi)
$$

We call this type of branes BPS branes. These branes allow solutions in the bosonic sector respecting 5 dimensional bulk+brane supersymmetry, the BPS condition preserves half of the supercharges corresponding to unbroken $\mathrm{N}=1$ supersymmetry in 4 
dimensions [15]. This type of models with exponential potentials can possibly arise from the bosonic sector of dimensionally reduced supergravity theory [11]. The localization of scalar modes were discussed with one brane in [16] based on $O(3)$ symmetry in the generalized longitudinal gauge of [17].

There is always a flat background solution for any value of $r_{c}$. The change of $r_{c}$ is described by a massless modulus field, the radion in the low energy effective theory. There is an additional dilatational invariance of the action with BPS branes leading to a second zero mode. The value of the scalar field on the Planck brane is an integration constant, a free parameter of the action. Choosing a definite background solution this symmetry is broken spontaneously and the corresponding massless Goldstone boson is expected in the scalar spectrum. In the limit of the Randall-Sundrum scenario this invariance is obvious and is presented in the next section.

To obtain an analytical solution for the KK modes the following particular potential will be used

$$
W(\Phi)= \pm \lambda_{i}(\Phi)=\frac{6 k}{\kappa^{2}} e^{\alpha \Phi} .
$$

The solution contains two integration constants $c_{0}, C_{P}$, usually both chosen to 1

$$
\begin{aligned}
a(y) & =c_{0}\left(C_{P}-\frac{3 \alpha^{2}}{\kappa^{2}} k y\right)^{\frac{\kappa^{2}}{3 \alpha^{2}}}, \\
e^{-\alpha \Phi_{0}(y)} & =\left(C_{P}-\frac{3 \alpha^{2}}{\kappa^{2}} k y\right) .
\end{aligned}
$$

In the limit $\alpha \rightarrow 0$ we get back the AdS $e^{-k y}$ profile of the Randall-Sundrum scenario and the scalar field decouples from gravity. We deal with solution with

$0<\alpha^{2}<\frac{\kappa^{2}}{3 k r_{c}}$ to avoid naked singularities in the bulk. This solution generates a hierarchy of $a\left(r_{c}\right)=\left(1-\frac{3 \alpha^{2}}{\kappa^{2}} k r_{c}\right)^{\frac{\kappa^{2}}{3 \alpha^{2}}}$. The dilatational invariance corresponds to the free choice of $C_{P}=e^{-\alpha \Phi_{0}(0)}$ the value of the scalar field on the Planck brane. It's change can be compensated by a change in $k, c_{0}$ to have the same warp factor $a(y)$ and superpotential. For particular solutions we use $c_{0}=C_{P}=1$. In the next section we discuss the special limit of the Randall-Sundrum scenario.

\section{$3 \quad$ RS1 with bulk scalar field}

In the RS1 scenario specially tuned bulk and brane cosmological constants provide a warped solution. In a more realistic model a dynamical mechanism is needed to produce the brane and bulk constants. A simple proposal is to add a bulk scalar field which has constant bulk and brane potentials. This is the limit of the previously presented model with

$$
\begin{aligned}
& W(\Phi)=\lambda(\Phi)=\frac{6 k}{\kappa^{2}}=\text { constant } \\
& V(\Phi)=\Lambda=-\frac{6 k^{2}}{\kappa^{2}} .
\end{aligned}
$$

The classical background solution

$$
a(y)=e^{-k|y|}, \quad \Phi_{0}(y)=\Phi_{R S}=\text { constant }
$$


has two invariances. One is related to the free choice of the distance $r_{c}$ between the two branes, the other is the freedom that we can shift the constant scalar field $\Phi_{R S}$ to $\Phi_{R S}+c_{1}$, it is also an exact solution with the same warp factor.

In the linearized theory the former provides the massless radion [4] the latter zero mode can be identified as follows. The linearized gravitational $\left(h_{a b}\right)$ and scalar $(\phi(x, y))$ perturbations around (19) decouple, it was also realized in [16],[20]. The gravitational perturbations are as in RS1, a massless radion with the wavefunction

$$
F(x, y)=-\frac{a^{\prime}}{a} \frac{1}{a^{2}} f(x)=k \frac{1}{a^{2}} f(x), \square f(x)=0,
$$

a massless spin-2 graviton localized on the Planck brane and its KK tower.

The scalar field perturbations are determined from

$$
\square_{5} \phi(x, y)=0,
$$

with the boundary condition $\phi^{\prime}\left(x, y_{i}\right)=0$. This is the free Klein-Gordon equations on an $\mathrm{AdS}_{5}$ background, with $\square=\square_{4}=\partial^{\mu} \partial_{\mu}$

$$
\square_{5}=-\partial_{z}^{2}-4 \frac{a^{\prime}}{a} \partial_{z}+\frac{1}{a^{2}} \square .
$$

The scalar field spectrum contains a dilaton zero mode

$$
\phi(x, y)=f_{1}(x), \quad \square f_{1}(x)=0,
$$

reflecting the invariance of the action against the shift of the constant value of the scalar field. The background solution brakes this symmetry spontaneously and this zero mode is the corresponding Goldstone boson. This zero mode mixes with gravitational perturbations if the linearized scalar equations are coupled to gravity.

We solve the equation for the KK modes in Schrödinger basis: change to conform flat background by $d y=a(z) d z$ with $a(z)=1 /(1+k|z|)$ and rescale the field by $\phi(x, z)=e^{i p x} \Phi_{0}^{\prime} a^{-\frac{3}{2}} \tilde{\phi}(z)$ also changing to mass eigenvectors with $\square \phi_{i}=-m_{i}^{2} \phi_{i}$.

$$
\tilde{\phi}^{\prime \prime}-\left(\frac{9}{4}\left(\frac{a^{\prime}}{a}\right)^{2}+\frac{3}{2}\left(\frac{a^{\prime}}{a}\right)^{\prime}\right) \tilde{\phi}+m^{2} \tilde{\phi}=0
$$

The boundary condition is $\left(a^{-\frac{3}{2}} \tilde{\phi}(z)\right)^{\prime}=0$. The potential term multiplying $\tilde{\phi}$ can be written as

$$
V(z)=\frac{\left(a^{\frac{3}{2}}\right)^{\prime \prime}}{a^{\frac{3}{2}}}=\frac{\left(4-\frac{1}{4}\right) k^{2}}{(1+k|z|)^{2}} .
$$

The massive solutions are determined by Bessel functions

$$
\tilde{\phi}=\sqrt{1+k|z|}\left(A J_{2}\left(\frac{m}{k}(1+k|z|)\right)+B Y_{2}\left(\frac{m}{k}(1+k|z|)\right)\right) .
$$

The ratio $A / B$ determined from one boundary condition the mass eigenvalues are determined at the other brane and are of the order of the TeV scale.

The scalar spectrum then contains two zero modes and a KK tower. Turning on smoothly the bulk and brane scalar field potentials continuous deformation of the spectrum is expected. Quantum corrections are also expected to develop a nontrivial bulk and brane potential for the scalar field. The linearized equations for the action (1) is presented in the next section. 


\section{Linear perturbations}

In this section we give the equation of motion for general linear perturbations keeping the branes at the fixed orbifold points and discuss the role of gauge freedom. A general perturbation is

$$
\begin{aligned}
& \delta g_{\mu \nu}=a^{2} h_{\mu \nu}(x, y), \quad \delta g_{5 \nu}=h_{5 \nu}(x, y) \\
& \delta g_{55}=h_{55}=-2 G(x, y), \\
& \delta \Phi=\phi(x, y) .
\end{aligned}
$$

Infinitesimal general coordinate transformations

$$
x^{5} \rightarrow x^{5}+\xi^{5}, \quad x^{\mu} \rightarrow x^{\mu}+\xi^{\mu},
$$

must fulfill the orbifold symmetry conditions, $\xi_{\mu}$ continuous and even, $\xi_{5}$ odd and vanishes at the branes.

$$
\begin{array}{r}
\xi_{\mu}\left(x, y+y_{i}\right)=\xi_{\mu}\left(x, y-y_{i}\right) \\
\xi_{5}\left(x, y+y_{i}\right)=-\xi_{5}\left(x, y-y_{i}\right)
\end{array}
$$

$\xi_{5}$ gauge transformations moving the orbifold fixed points are not allowed in this approach. The infinitesimal change of the background solution is understood as a change in the perturbations.

$$
\begin{aligned}
\delta h_{\mu \nu} & =\left(\xi_{\mu, \nu}+\xi_{\nu, \mu}\right)-2 \frac{a^{\prime}}{a} \xi_{5} \eta_{\mu \nu} \\
\delta h_{5 \mu} & =\xi_{5, \mu}+a^{2} \xi_{\mu, 5} \\
\delta h_{55} & =2 \xi_{5}^{\prime} . \\
\delta \phi & =-\Phi_{0}^{\prime} \xi_{5}
\end{aligned}
$$

The offdiagonal $h_{5 \mu}$ components can be transformed out with

$$
\xi_{\mu}=-\int d y \frac{1}{a^{2}(y)} h_{5 \mu}, \quad \xi_{5}=0 .
$$

The branes are not moved in the $5^{\text {th }}$ dimension.

Without loss of generality the Einstein equation can be used without offdiagonal linear perturbation. The metric is

$$
d s^{2}=a^{2}(y)\left(\eta_{\mu \nu}+h_{\mu \nu}(x, y)\right) d x^{\mu} d x^{\nu}-(1+2 G(x, y)) d z^{2} .
$$

(31) was our first gauge choice and there is a restricted gauge degree of freedom to keep $h_{5 \mu}=0 . \xi_{5}(x, y)$ is constrained by the orbifold conditions (30) and

$$
\xi_{\mu}(y, x)=-\int_{0}^{y} d y_{1} \frac{1}{a^{2}\left(y_{1}\right)} \xi_{5, \mu}\left(x, y_{1}\right)+\epsilon_{\mu}(x) .
$$

In what follows we work on the interval $\left(0, r_{c}\right)$ and get the fields on the orbifold using the $Z_{2}$ symmetry.

The linearized equations are the following, '55', ' $5 \mu$ ' and ' $\mu \nu$ ' respectively.

$$
\begin{aligned}
\frac{\square G}{a^{2}}+4 \frac{a^{\prime}}{a} G^{\prime} & +2\left(4 \frac{a^{\prime 2}}{a^{2}}+\left(\frac{a^{\prime}}{a}\right)^{\prime}\right) G-\frac{1}{2 a^{2}}\left(a^{2} h^{\prime}\right)^{\prime}= \\
& \kappa^{2}\left[\frac{2}{3}\left(\Phi_{0}^{\prime \prime}+4 \frac{a^{\prime}}{a} \Phi_{0}^{\prime}\right) \phi+2 \Phi_{0}^{\prime} \phi^{\prime}+\frac{2}{3} \sum_{i}\left(\frac{\partial \lambda_{i}}{\partial \Phi} \phi+\lambda_{i} G\right) \delta\left(y-y_{i}\right)\right] .
\end{aligned}
$$




$$
\begin{gathered}
3 \frac{a^{\prime}}{a} G_{, \mu}+\frac{1}{2}\left(h_{\mu, \alpha}^{\alpha}-h_{, \mu}\right)^{\prime}=\kappa^{2} \Phi_{0}^{\prime}(y) \phi_{, \mu}(x, y) \\
-G_{, \mu \nu}-\quad\left[a^{\prime} a G^{\prime}+2\left(4 a^{\prime 2}+a^{2}\left(\frac{a^{\prime}}{a}\right)^{\prime}\right) G\right] \eta_{\mu \nu}+\frac{1}{2 a^{2}}\left(a^{4} h_{\mu \nu}^{\prime}\right)^{\prime}+\frac{1}{2} \frac{a^{\prime}}{a} h^{\prime} a^{2} \eta_{\mu \nu}+ \\
+\frac{1}{2}\left(2 h_{(\mu, \nu) \lambda}^{\lambda}-h_{\mu \nu, \lambda}^{, \lambda}-h_{, \mu \nu}\right)= \\
-\frac{2 \kappa^{2}}{3} a^{2} \eta_{\mu \nu}\left(\Phi_{0}^{\prime \prime}+4 \frac{a^{\prime}}{a} \Phi_{0}^{\prime}\right) \phi+\frac{\kappa^{2}}{3} \sum_{i} a^{2} \eta_{\mu \nu}\left(\frac{\partial \lambda_{i}}{\partial \Phi} \phi+\lambda_{i} G\right) \delta\left(y-y_{i}\right)
\end{gathered}
$$

Here $h=h_{\mu}^{\mu}, \square=\partial^{\mu} \partial_{\mu}$. The scalar equation is

$$
\begin{aligned}
& \frac{1}{a^{2}} \square \phi-\phi^{\prime \prime}-4 \frac{a^{\prime}}{a} \phi^{\prime}+\frac{\mathrm{d}^{2} V}{\mathrm{~d} \Phi^{2}} \phi= \\
& \left(\frac{1}{2} h^{\prime}-G^{\prime}\right) \Phi_{0}^{\prime}-2 G \frac{\mathrm{d} V}{\mathrm{~d} \Phi}-\sum_{i}\left(\frac{\mathrm{d}^{2} \lambda_{i}}{\mathrm{~d} \Phi^{2}} \phi+G \frac{\mathrm{d} \lambda_{i}}{\mathrm{~d} \Phi}\right) \delta\left(y-y_{i}\right) .
\end{aligned}
$$

The singular terms at the branes define boundary conditions. The complete $5 \mu$ equation is valid also at the branes. The 55 boundary condition is the trace of $\mu \nu$ boundary condition calculated from (36). It is equivalent to the Israel jump equation calculated with the extrinsic curvature. From $\mu \nu(36)$ we get

$$
h_{\mu \nu}^{\prime}-2 \frac{a^{\prime}}{a} G(x, y) \eta_{\mu \nu}+\frac{2 \kappa^{2}}{3} \Phi_{0}^{\prime} \phi \eta_{\mu \nu}=0 \text { at } y=y_{i}
$$

Already the leading order boundary conditions and $Z_{2}$ symmetry were used. The scalar equation gives

$$
\pm 2 \phi^{\prime}\left(x, y_{i}\right)=\frac{\mathrm{d}^{2} \lambda_{i}}{\mathrm{~d} \Phi^{2}} \phi\left(x, y_{i}\right)+\frac{\mathrm{d} \lambda_{i}}{\mathrm{~d} \Phi} G\left(x, y_{i}\right)
$$

The equations are not idependent. The Bianchi identities ensure that from the 15 Einstein equations are only 10 independent and the bulk scalar equation can be derived from the Einstein equations with differentiation and taking linear combinations. The scalar boundary condition presents a meaningful constraint. The equation are solved for special BPS branes fulfilling (13).

\subsection{Lorentz decomposition of $h_{\mu \nu}$}

To solve the equations we decompose $h_{\mu \nu}$ in a Lorentz covariant way. It contains a TT (transverse traceless) symmetric tensor ( 5 components), one divergencefree vector (3) and two scalars (1+1) giving 10 components.

$$
h_{\mu \nu}=\tilde{h}_{\mu \nu}(x, y)+A_{\mu, \nu}(x, y)+A_{\nu, \mu}(x, y)+\partial_{\mu} \partial_{\nu} b(x, y)-F(x, y) \eta_{\mu \nu}
$$

Here

$$
\begin{aligned}
& \tilde{h}_{\mu, \nu}^{\nu}=0, \quad \tilde{h}_{\mu}^{\mu}=0, \\
& A_{, \mu}^{\mu}=0 .
\end{aligned}
$$


The components can be uniquely defined if the perturbations are assumed to be Lorentz covariant as the background solution is Lorentz invariant and fulfill

$$
\square A_{\mu} \neq 0, \quad \square b \neq 0,
$$

to have nonvanishing trace or divergence in $h_{\mu \nu}$. They are defined by

$$
\begin{aligned}
\square^{2} b & =\frac{4}{3}\left(\partial_{\alpha} \partial_{\beta}-\square \frac{1}{4} \eta_{\alpha \beta}\right) h^{\alpha \beta}, \\
F & =\frac{1}{4}(-h+\square b), \\
\square A_{\mu} & =\left(h_{\mu, \lambda}^{\lambda}+F_{, \mu}-\square b_{, \mu}\right) .
\end{aligned}
$$

This sequential definition of components ensures that $F, \square b$ describe the complete trace and $F, b, \square A_{\mu}$ describe the whole divergence of $h_{\mu \nu} .{ }^{3}$ The TT part is defined as the vector and scalar parts subtracted from $h_{\mu \nu} . \tilde{h}_{\mu \nu}$ can still contain scalar and vector TT combinations but those will be proved to be gauge degrees of freedom as in four dimensional gravity.

Gauge transformations of the components can be defined as

$$
\begin{aligned}
\delta \phi & =-\Phi_{0}^{\prime} \xi_{5}(x, y), \\
\delta G & =-\xi_{5}(x, y)^{\prime}, \\
\delta F & =2 \frac{a^{\prime}}{a} \xi_{5}(x, y) .
\end{aligned}
$$

The last two transformation can be used to fix $\xi_{5}(x, y)$ without moving the branes and setting the $F(x, y)-G(x, y)$ difference only $x$ dependent. Make a gauge transformation with

$$
\begin{aligned}
a^{2} \xi_{5}(x, y) & =-\int_{0}^{y} a^{2}\left(y_{1}\right)\left(F\left(x, y_{1}\right)-G\left(x, y_{1}\right)\right) d y_{1}+\chi(y) \int_{0}^{r_{c}} a^{2}(F-G) d y_{1}, \\
\chi(y) & =\frac{\int_{0}^{y} a^{2}\left(y_{1}\right) d y_{1}}{\int_{0}^{r_{c}} a^{2}\left(y_{1}\right) d y_{1}} .
\end{aligned}
$$

This $\xi_{5}$ fulfills the orbifold condition and it is completely fixed. The gauge transformation was motivated by the work of Kubyshin et al. who have found the radion wave function with fixed flat branes [14]. In the new gauge

$$
F^{n}(x, y)-G^{n}(x, y)=f_{\Delta}(x)=\frac{1}{\int_{0}^{r_{c}} a^{2}\left(y_{1}\right) d y_{1}} \int_{0}^{r_{c}} a^{2}(F-G) d y_{1}
$$

We work in this gauge and drop the $n$ index in what follows. The remaining gauge degree of freedom is

$$
\xi_{\mu}=\epsilon_{\mu}(x),
$$

which can be only used to set the two helicity state of the massless four dimensional graviton. The gauge transformation of the other components is

$$
\begin{aligned}
\square \delta b & =2 \epsilon_{, \mu}^{\mu}, \text { if } \square \epsilon_{, \mu}^{\mu} \neq 0 \\
\delta A_{\mu} & =\epsilon_{\mu}, \text { if } \square \epsilon_{\mu} \neq 0, \epsilon_{, \mu}^{\mu}=0
\end{aligned}
$$

\footnotetext{
${ }^{3} \mathrm{~A}$ subtlety of the decomposition that $h_{\mu \nu}$ only defines $\square^{2} b$ while in the definition of $F, A_{\mu}$ purely $\square b$ appears.
} 
Untill this point the equations of motion were not used and the gauge could be partially fixed. In what follows we use the Einstein equations and show that $A_{\mu}(x, y), b(x, y)$ can be gauged away and $\tilde{h}_{\mu \nu}$ contains only the graviton and its KK tower. The single $F(x, y)$ field will describe the massless radion and the scalar field together.

\subsection{Solving the Einstein equations}

The equations can be written up with the general decomposition of $h_{\mu \nu}$. The $5 \mu$ equation (35) gives

$$
\frac{1}{2} \square A_{\mu}^{\prime}=\kappa^{2} \Phi_{0}^{\prime}(y) \phi_{, \mu}(x, y)-\frac{3}{2} F_{, \mu}^{\prime}-3 \frac{a^{\prime}}{a} G_{, \mu} .
$$

Different Lorentz spin perturbations decouple and we get

$$
A_{\mu}^{\prime}(x, y)=0
$$

as it was defined as $\square A_{\mu} \neq 0$. The solution is

$$
A_{\mu}(x, y)=f_{\mu}(x), \quad f_{, \mu}^{\mu} \neq 0, \square f_{\mu}(x) \neq 0 .
$$

It can be gauged to nothing with

$$
\epsilon_{\mu}(x)=-f_{\mu}(x) .
$$

The RHS of (54) gives setting one y-dependent integration constant zero

$$
\frac{2 \kappa^{2}}{3} a^{2} \Phi_{0}^{\prime} \phi=\left(a^{2} G\right)^{\prime} .
$$

In the $\mu \nu$ Einstein equation (36) as the result of assumed Lorentz covariance the $\partial_{\mu} \partial_{\nu}$ derivatives terms decouple from the TT and $\eta_{\mu \nu}$ part and give the equation

$$
\frac{1}{a^{2}}(F-G)_{, \mu \nu}+\frac{1}{2 a^{2}}\left(a^{4} b_{, \mu \nu}^{\prime}\right)^{\prime}=0 .
$$

The general solution sending two integration constant in the infinite $x$ to zero is

$$
b(x, y)=b_{1}(x)+b_{2}(x) \int \frac{1}{a^{4}}+2 f_{\Delta}(x) \int \frac{1}{a^{4}} \int a^{2}, \text { if } \square f_{\Delta}(x) \neq 0 .
$$

The trace of the $\mu \nu$ boundary condition (38) reads

$$
\square b^{\prime}-4\left(F^{\prime}+2 \frac{a^{\prime}}{a} G-\frac{2 \kappa^{2}}{3} a^{2} \Phi_{0}^{\prime} \phi\right)=0 .
$$

The (58) solution of $5 \mu$ is also valid at the branes giving with $\square b \neq 0$

$$
b^{\prime}\left(x, y_{i}\right)=0 .
$$

At $y=0$ it sets $b_{2}(x)=0$ and at $r_{c} f_{\Delta}(x)=0$. The remaining solution in $b(x, y)$ can be gauged to zero by

$$
\epsilon_{, \lambda}^{\lambda}(x)=-\frac{1}{2} b_{1}(x)
$$


If $\square f_{\Delta}(x)=0$ then using the TT part of the ' $\mu \nu$ ' equation, one gets $f_{\Delta}(x)_{, \mu \nu}=0$ and $f_{\Delta}(x)=0$.

We proved using the Lorentz covariance of the linear perturbations that the following gauge can always be chosen. There is a TT tensor and a single $F(x, y)$ scalar perturbation $(F=G)$.

$$
\begin{aligned}
h_{\mu \nu} & =\tilde{h}_{\mu \nu}-F(x, y) \eta_{\mu \nu} \\
h_{55} & =-2 F(x, y) \\
\frac{2 \kappa^{2}}{3} \Phi_{0}^{\prime} \phi & =F^{\prime}(x, y)+2 \frac{a^{\prime}}{a} F(x, y)
\end{aligned}
$$

This gauge is called Newton gauge by Tanaka and Montes in [9] and was used in [10]. To our knowledge this gauge choice is correct in the presence of two BPS branes. The remaining gauge degree of freedom, $\square \epsilon_{\mu}(x)=0$ can transform out the 3 non-physical component of the massless graviton.

The scalar and the spin-2 TT perturbation decouple. The TT equation is

$$
\frac{1}{2 a^{2}}\left(a^{4} \tilde{h}_{\mu \nu}^{\prime}\right)^{\prime}-\frac{1}{2} \square h_{\mu \nu}^{T T}=0,
$$

together with the boundary condition

$$
\tilde{h}_{\mu \nu}^{\prime}\left(x, y_{i}\right)=0 .
$$

The boundary condition only allow the massless solution of

$$
\tilde{h}_{\mu \nu}=h_{\mu \nu}^{G}(x)
$$

the massless graviton with 2 helicity states. It is localized at the Planck brane with larger warp factor. There is also a massive graviton KK tower, with 5 degrees of freedom. The KK modes are not localized.

Upon using the Newton gauge relation (66) the Einstein boundary conditions automatically fulfilled. In the bulk the scalar part of the Einstein and the KleinGordon equations are equvalent.

$$
\frac{1}{2 a^{2}} \square F-\frac{1}{2} F^{\prime \prime}+\left(\frac{\Phi_{0}^{\prime \prime}}{\Phi_{0}^{\prime}}-\frac{a^{\prime}}{a}\right) F^{\prime}+2\left(\left(\frac{a^{\prime}}{a}\right) \frac{\Phi_{0}^{\prime \prime}}{\Phi_{0}^{\prime}}-\left(\frac{a^{\prime}}{a}\right)^{\prime}\right) F=0
$$

This is accompanied by the scalar boundary condition (39). Eliminating $\phi(x, y)$ it is

$$
\left(a^{2} F\right)^{\prime \prime}-\left(2 \frac{a^{\prime}}{a}+\frac{\Phi_{0}^{\prime \prime}}{\Phi_{0}^{\prime}} \pm \frac{1}{2} \frac{\mathrm{d}^{2} \lambda_{i}}{\mathrm{~d} \Phi^{2}}\right)\left(a^{2} F\right)^{\prime}+2\left(\frac{a^{\prime}}{a}\right)^{\prime}\left(a^{2} F\right)=0 .
$$

This boundary condition contains a second derivative, it makes generally the operator in the radion differential equation non-hermitic and causes the hermiticity problem observed in [10]. The different mass eigenmodes are not orthogonal to each other and does not serve as a basis for expanding a general solution. The bulk equation can be used to eliminate the second derivative but then the d'Alembertian appears with different eigenvalues for different eigenfunctions. This simplified boundary condition is

$$
\left(\frac{\Phi_{0}^{\prime \prime}}{\Phi_{0}^{\prime}} \pm \frac{1}{2} \frac{\mathrm{d}^{2} \lambda_{i}}{\mathrm{~d} \Phi^{2}}\left(\Phi_{0}\right)\right)\left(a^{2} F\right)^{\prime}+\square(F)=0 \quad \text { at } y=0, r_{c} .
$$


It is generally still not hermitic, though with BPS branes the first term vanishes as $\frac{\Phi_{0}^{\prime \prime}}{\Phi_{0}^{\prime}}=\frac{1}{2} \frac{\mathrm{d}^{2} W}{\mathrm{~d} \Phi^{2}}$. The

$$
\square F\left(x, y_{i}\right)=0
$$

boundary condition defines a hermitic operator for massive perturbations. Practically there is no boundary condition for the massless solutions.

\subsection{Massless scalar perturbations}

There are two independent massless modes as the boundary condition (73) is automatically fulfilled. The radion differential equation can be written as

$$
\frac{\left(\frac{a^{\prime}}{a}\right)^{\prime}}{\left(\frac{a^{\prime}}{a}\right)}\left(\frac{1}{a^{2}} \frac{\left(\frac{a^{\prime}}{a}\right)^{2}}{\left(\frac{a^{\prime}}{a}\right)^{\prime}}\left(a^{2} \frac{1}{\frac{a^{\prime}}{a}} F\right)^{\prime}\right)^{\prime}-\frac{\square}{a^{2}} F=0
$$

The wavefunction of the massless radion is

$$
F_{R}(x, y)=F_{R}(y) r(x)=\epsilon(y) \frac{a^{\prime}}{a} \frac{1}{a^{2}(y)} r(x) \quad \square r(x)=0,
$$

where $\epsilon(y)$ is the sign function and it makes the radion wavefunction even under $Z_{2}$. The radion appears in the scalar field perturbation as $\phi_{R}(x, y)=-\frac{\Phi_{0}^{\prime}}{2 a^{2}} r(x)$. In the limit of no scalar field (75) gives back the result of Charmousis et al. [4] $F_{R}=-k e^{2 k y} r(x)$. The radion describes the relative free motion of the branes. With a gauge transformation of

$$
\xi_{5}(x, y)=-\frac{1}{2 a^{2}} r(x),
$$

the radion solution is completely transformed to a scalar TT mode and the relative motion of the branes. Keeping $h_{5 \mu}=0$ with (33) after this gauge transformation the bulk metric is

$$
\begin{aligned}
h_{\mu \nu} & =\tilde{h}_{\mu \nu}+r_{, \mu \nu}(x) \int_{0}^{y} \frac{1}{a^{4}}-F_{s}(x, y) \eta_{\mu \nu} \\
h_{55} & =-2 F_{s}(x, y) \\
\frac{2 \kappa^{2}}{3} \Phi_{0}^{\prime} \phi_{s} & =F_{s}^{\prime}(x, y)+2 \frac{a^{\prime}}{a} F_{s}(x, y) .
\end{aligned}
$$

$F_{s}, \phi_{s}$ here only describes the scalar field perturbations. With BPS branes this gauge transformation is well defined and the relative motion of the branes is

$$
\Delta r_{c}=\frac{1}{2}\left(\frac{1}{a^{2}(0)}-\frac{1}{a^{2}\left(r_{c}\right)}\right) r(x) .
$$

This single radion solution was analyzed in [12] without the dynamics of the scalar field.

The scalar field has a zero mode and a complete KK tower. The even massless scalar solution of (74) with flat branes is

$$
F_{D}(x, y)=F_{D}(y) f_{1}(x)=\frac{a^{\prime}}{a} \frac{1}{a^{2}(y)} f_{1}(x) \int_{0}^{y} \mathrm{~d} y_{1} a^{2} \frac{\left(\frac{a^{\prime}}{a}\right)^{\prime}}{\left(\frac{a^{\prime}}{a}\right)^{2}} \quad, \quad \square f_{1}(x)=0 .
$$


This is the dilatonic zero mode of the scalar field, it is the Goldstone boson of the spontaneously broken dilatational invariance of the action. It appeared as adjusting a meaningless constant in the limit of the Randall-Sundrum scenario with decoupled scalar field. Brax et al. did not discuss this second zero mode in [12]. They were aware of two scalar zero modes [18] and recently studied the cosmological evolution of them [19].

The extra dilatonic zero mode given in (81) vanishes on the Planck brane and its wavefunction is the radion wavefunction multiplied with an extra y-function. The two solutions are not orthogonal to each other, they can be orthogonalized with Gram-Schmidt method, once a scalar product is defined on the interval $\left(0, r_{c}\right)$. Two solutions can be distinguished also on physical grounds. The dilatonic zero mode (81) also describes a relative motion of the branes through changing the proper distance $\int_{0}^{r_{c}} \sqrt{\left|g_{55}\right|} \mathrm{d} y=r_{c}+\int_{0}^{r_{c}} F(x, y) \mathrm{d} y$. A new dilaton wavefunction can be formed with linear combination that keeps the proper distance $r_{c}$ unchanged.

$$
F_{2}(y)=F_{D}(y)-\frac{\int_{0}^{r_{c}} F_{D}(y)}{\int_{0}^{r_{c}} F_{R}(y)} F_{R}(y)
$$

Now the two massless modes have different physical origin as one moves the branes the other not. The radion orthogonal to $F_{2}(y)$ with respect to a specific scalar product can be defined as $\tilde{F}_{R}(y)=F_{R}(y)-F_{2}(y)\left(F_{R}, F_{2}\right) /\left(F_{2}, F_{2}\right)$.

The dilatonic zero mode remarkably simplify with exponential superpotential. Performing the integral in the solution (81) we get

$$
F_{D}(x, y)=-f_{1}(x) \frac{1}{1+\frac{2 \kappa^{2}}{3 \alpha^{2}}}\left(\frac{1}{k} \frac{a^{\prime}}{a} \frac{1}{a^{2}}+1\right) \simeq f_{1}(x)\left(F_{R}(y)-F_{R}(0)\right) .
$$

Subtracting the radion part we get $F_{D}(x, y)=f_{1}(x) /\left(1+\frac{2 \kappa^{2}}{3 \alpha^{2}}\right)$ which implies via (66) the dilaton scalar field solution

$$
\phi_{D}(x, y)=-\frac{1}{\alpha} \frac{1}{1+\frac{2 \kappa^{2}}{3 \alpha^{2}}} f_{1}(x) .
$$

In the limit $\alpha \rightarrow 0$ it is the dilaton of (23). This solution can be found directly as the coefficient of the term $F$ in the radion equation (70) vanishes for $W \sim e^{\alpha \Phi}$,

$$
\left(\left(\frac{a^{\prime}}{a}\right) \frac{\Phi_{0}^{\prime \prime}}{\Phi_{0}^{\prime}}-\left(\frac{a^{\prime}}{a}\right)^{\prime}\right)=\frac{\kappa^{2}}{12} W^{2}\left(\frac{W^{\prime}}{W}\right)^{\prime}=0, \text { here } W^{\prime}=\frac{\partial W}{\partial \Phi} .
$$

Thus the equation (70) has a $y$ independent $F_{D}(x, y) \simeq f_{1}(x)$ dilaton solution of (84).

The dilatonic zero mode, called $\omega_{2}$ was found by Bozza et al. [16] with one brane in the case of dilatonic superpotential. This was the only propagating massless mode as the radion $\omega_{3}$ is not normalizable and not physical with one brane. $\omega_{2}$ was identified as the dilaton in the limit of the RS1 scenario when the scalar field decouples from gravity. The Einstein equations gave constraints between the massive modes and there were two independent scalar KK tower. One is the scalar field KK tower. The other completes the two component transverse traceless tensor $\bar{h}_{i j}$ with two additional 3-vector mode to a 5 componenent massive 4-dimensional KK graviton. Bozza et al. investigated the scalar perturbation of a Lorentz invariant setup based 
on 3 dimensional spatial covariance. At the end 4 dimensional covariant equations described the perturbations. It would be enlightening to perform their analysis in 4 dimensional language and understand the physics of perturbations. Indeed following their method with two branes we also got two massless scalar modes. This work is in progress.

\subsection{Massive KK tower}

There is a KK tower of the massive scalar field perturbations with the well defined hermitic boundary conditions $F\left(x, y_{i}\right)=0$. In a scenario with small backreaction like in [10], the KK spectrum can be solved with good approximation using the exponential warp factor $a(y)=e^{-k y}$. The mass eigenvalues determined by the zeros of the Bessel functions and are at the order of the weak scale $\left(k e^{-k r_{0}}\right)$.

The radion equation (70) for the case of exponential superpotential (14) can be analytically solved by Bessel functions. As a standard technique we change to conform flat background by $d y=a(z) d z$ and rescale the field $\mathrm{F}$ by $F(x, z)=$ $e^{i p x} \Phi_{0}^{\prime} a^{-\frac{3}{2}} \tilde{F}(z)$ also changing to mass eigenvectors with $\square F_{i}=-m_{i}^{2} F_{i}$. The radion equation in this Schrödinger basis is

$$
\tilde{F}^{\prime \prime}-\left(\frac{9}{4}\left(\frac{a^{\prime}}{a}\right)^{2}-\frac{5}{2}\left(\frac{a^{\prime}}{a}\right)^{\prime}+\frac{a^{\prime}}{a} \frac{\Phi_{0}^{\prime \prime}}{\Phi_{0}^{\prime}}+2\left(\frac{\Phi_{0}^{\prime \prime}}{\Phi_{0}^{\prime}}\right)^{2}-\frac{\Phi_{0}^{\prime \prime \prime}}{\Phi_{0}^{\prime \prime}}\right) \tilde{F}+m^{2} \tilde{F}=0
$$

This equation for each eigenmode has a mass parameter and two integration constants. One integration constant is fixed by the normalization, the other from the boundary condition at the Planck brane $(y=0)$ and the mass is determined from the $\mathrm{TeV}$ brane $\left(r_{c}\right)$ boundary condition. With the exponential superpotential the equation is

$$
\tilde{F}^{\prime \prime}+\left(m^{2}-\frac{\nu^{2}-\frac{1}{4}}{\zeta^{2}}\right) \tilde{F}=0
$$

where

$$
\begin{aligned}
\zeta & =\frac{1}{k\left(1-\frac{3 \alpha^{2}}{\kappa^{2}}\right)}+z=\frac{1}{\tilde{k}}+z, \\
\nu & =-\frac{1}{2}-\frac{3}{2} \frac{1}{\frac{3 \alpha^{2}}{\kappa^{2}}-1} .
\end{aligned}
$$

The range of $\nu$ is $1 \leq \nu<\infty, \nu=1$ corresponding to the RS scenario. The solution fulfilling the $y=z=0$ boundary condition is given by two type of Bessel functions

$$
\tilde{F}=\sqrt{\zeta}\left(Y_{\nu}\left(\frac{m}{\tilde{k}}\right) J_{\nu}(m \zeta)-J_{\nu}\left(\frac{m}{\tilde{k}}\right) Y_{\nu}(m \zeta)\right)
$$

The mass eigenvalues are determined at $y=r_{c}$ from the equation

$$
J_{\nu}\left(\frac{m_{i}}{\tilde{k}}\right) \frac{Y_{\nu}\left(m_{i} \zeta_{c}\right)}{Y_{\nu}\left(\frac{m_{i}}{\tilde{k}}\right)}-J_{\nu}\left(m_{i} \zeta_{c}\right)=0
$$

with

$$
\zeta_{c}=\frac{1}{\tilde{k}}\left(1-\frac{3 \alpha^{2}}{\kappa^{2}} k r_{c}\right)^{\frac{\kappa^{2}}{3 \alpha^{2}}}=\frac{a\left(r_{c}\right)}{\tilde{k}}
$$


There is always an $m_{i}=0$ eigenvalue, a massless solution. When the warp factor generates a large hierarchy between the two branes $a\left(r_{c}\right) \ll a(0)=1$ then the lowest mass eigenvalues are practically determined from the second term, the zeros of the Bessel function $J_{\nu}\left(m_{i} \zeta_{c}\right)$. The lowest $\mathrm{KK}$ masses are few time the warped mass scale $\tilde{k} a^{-1}\left(r_{c}\right)$, the $\mathrm{TeV}$ scale.

$\tilde{F}$ uniquely defines $\tilde{\phi}$ via $(66)$ and

$$
\tilde{\phi} \simeq\left(\frac{6 \alpha^{2}}{\kappa^{2} m} \frac{1}{\sqrt{\zeta}} J_{\nu}(m \zeta)-\left(1+\frac{3 \alpha^{2}}{\kappa^{2}}\right) J_{1+\nu}(m \zeta)\right) Y_{\nu}\left(\frac{m}{k}\right)-(Y \leftrightarrow J)
$$

In the limit $\alpha=0, \nu=1$ we get back the masive spectrum given in (26).

\section{Conclusion}

In this paper we have analyzed the coupled scalar gravitational system in 5 dimensions. Around an exact background solution we carefully fixed the gauge and solved the 5 dimensional linear equations of motion on the orbifold. We have found that with the special BPS brane potentials there are two scalar zero modes and one KK tower. The two scalar zero modes are the radion and the dilaton which can be identified in the smooth limit of the RS1 scenario with a free bulk scalar field. Both modes couple to the matter on the $\mathrm{TeV}$ brane via the induced metric. The two massless scalar modes generally lead to unacceptable phenomenology, the radius is not stabilized. Cosmological evolution of two scalar moduli in this model were discussed recently [19]. However the BPS brane scenario may help us to find the correct linear spectrum in the Goldberger-Wise stabilization mechanism. The gauge fixing discussed in this paper and used in [10] [9] leads to non-hermitic differential operator for scalar perturbations for general brane potentials. The GW bulk scalar with detuned potential gives mass not just to the radion but also to the dilaton mode. It would be very interesting to follow the fate of these two modes and relating them to the first scalar KK mode. Effective theory and AdS/CFT based calculation so far could only capture the radion mode [8], [21], [22]. The possible resolution of the hermiticity problem is that a mode is droped during solving the Einstein equations which has the the detuning of the brane potentials as a source. This work is in progress.

\section{Acknowledgement}

The author is grateful to Zoltán Kunszt, Csaba Csáki and Martin Puchwein for useful conversation. This work was supported in part by SNF and OTKA 029803.

\section{References}

[1] L. Randall and R. Sundrum, Phys. Rev. Lett. 83 (1999) 4690 [hep-th/9906064].

L. Randall and R. Sundrum, Phys. Rev. Lett. 83 (1999) 3370 [hep-ph/9905221].

[2] H. Verlinde, Nucl. Phys. B 580, 264 (2000) [arXiv:hep-th/9906182].

[3] S. B. Giddings, S. Kachru and J. Polchinski, arXiv:hep-th/0105097. 
[4] C. Charmousis, R. Gregory and V. A. Rubakov, Phys. Rev. D 62 (2000) 067505 [hep-th/9912160].

[5] C. Csaki, M. Graesser, L. Randall and J. Terning, Phys. Rev. D 62, 045015 (2000) [arXiv:hep-ph/9911406].

[6] J. Garriga and T. Tanaka, Phys. Rev. Lett. 84 (2000) 2778 [hep-th/9911055].

[7] W. D. Goldberger and M. B. Wise, Phys. Rev. Lett. 83 (1999) 4922 [hep$\mathrm{ph} / 9907447]$.

[8] W. D. Goldberger and M. B. Wise, Phys. Lett. B 475, 275 (2000) [arXiv:hep$\mathrm{ph} / 9911457]$.

[9] T. Tanaka and X. Montes, Nucl. Phys. B 582 (2000) 259 [hep-th/0001092].

[10] C. Csaki, M. L. Graesser and G. D. Kribs, Phys. Rev. D 63 (2001) 065002 [hep-th/0008151].

[11] M. Cvetic, H. Lu and C. N. Pope, Phys. Rev. D 63, 086004 (2001) [arXiv:hepth/0007209].

[12] P. Brax, C. van de Bruck, A. C. Davis and C. S. Rhodes, Phys. Lett. B 531, 135 (2002) [arXiv:hep-th/0201191].

[13] O. DeWolfe, D. Z. Freedman, S. S. Gubser and A. Karch, Phys. Rev. D 62 (2000) 046008 [arXiv:hep-th/9909134].

[14] E. E. Boos, Y. A. Kubyshin, M. N. Smolyakov and I. P. Volobuev, Class. Quant. Grav. 19, 4591 (2002) [arXiv:hep-th/0202009].

[15] E. Bergshoeff, R. Kallosh and A. Van Proeyen, JHEP 0010, 033 (2000) [arXiv:hep-th/0007044];

A. Falkowski, Z. Lalak and S. Pokorski, Nucl. Phys. B 613, 189 (2001) [arXiv:hep-th/0102145].

[16] V. Bozza, M. Gasperini and G. Veneziano, Nucl. Phys. B 619, 191 (2001) [arXiv:hep-th/0106019].

[17] C. van de Bruck, M. Dorca, R. H. Brandenberger and A. Lukas, Phys. Rev. D 62, 123515 (2000) [arXiv:hep-th/0005032].

[18] P. Brax, C. v. Bruck, A. C. Davis and C. S. Rhodes, private communication.

[19] P. Brax, C. v. Bruck, A. C. Davis and C. S. Rhodes, arXiv:hep-th/0209158.

[20] A. Lewandowski and R. Sundrum, Phys. Rev. D 65, 044003 (2002) [arXiv:hepth/0108025].

[21] P. Kanti, K. A. Olive and M. Pospelov, Phys. Lett. B 538, 146 (2002) [arXiv:hep-ph/0204202];P. Kanti, S. Lee and K. A. Olive, arXiv:hep-ph/0209036.

[22] R. Rattazzi and A. Zaffaroni, JHEP 0104, 021 (2001) [arXiv:hep-th/0012248]. 